\title{
Wide field astronomical image compensation with multiple laser-guided adaptive optics
}

\author{
Michael Hart, N. Mark Milton, Christoph Baranec, Thomas Stalcup, Keith Powell, Eduardo \\ Bendek, Don McCarthy, and Craig Kulesa \\ Center for Astronomical Adaptive Optics, The University of Arizona, Tucson, AZ 85721 \\ ${ }^{*}$ California Institute of Technology, 1200 E. California Blvd., Pasadena, CA 91125
}

\begin{abstract}
We report closed-loop results obtained from the first adaptive optics system to deploy multiple laser guide beacons. The system is mounted on the $6.5 \mathrm{~m}$ MMT telescope in Arizona, and is designed to explore advanced altitude-conjugated techniques for wide-field image compensation. Five beacons are made by Rayleigh scattering of laser beams at $532 \mathrm{~nm}$ integrated over a range from 20 to $29 \mathrm{~km}$ by dynamic refocus of the telescope optics. The return light is analyzed by a unique Shack-Hartmann sensor that places all five beacons on a single detector, with electronic shuttering to implement the beacon range gate. Wavefront correction is applied with the telescope's unique deformable secondary mirror. The system has now begun operations as a tool for astronomical science, in a mode in which the boundary-layer turbulence, close to the telescope, is compensated. Image quality of 0.2-0.3 arc sec is routinely delivered in the near infrared bands from 1.2-2.5 $\mu \mathrm{m}$ over a field of view of 2 arc min. Although it does not reach the diffraction limit, this represents a 3 to 4 -fold improvement in resolution over the natural seeing, and a field of view an order of magnitude larger than conventional adaptive optics systems deliver. We present performance metrics including images of the core of the globular cluster M3 where correction is almost uniform across the full field. We describe plans underway to develop the technology further on the twin $8.4 \mathrm{~m}$ Large Binocular Telescope and the future $25 \mathrm{~m}$ Giant Magellan Telescope.
\end{abstract}

Keywords: Telescopes, adaptive optics, laser guide stars

\section{INTRODUCTION}

The $6.5 \mathrm{~m}$ MMT telescope on Mt. Hopkins in Arizona, and its predecessor the six-mirror Multiple Mirror Telescope, have for decades supported research into leading-edge high resolution imaging techniques, in particular adaptive optics (AO). The AO system now in place on the telescope continues that tradition and is in many ways not merely 'unconventional' but-so far-unique. In this paper we explore new results from the system that demonstrate a powerful new way to obtain high resolution over a substantially wider field of view (FOV) than is achievable with conventional AO systems. These results build on a number of innovations pioneered at the MMT:

- The AO system's wavefront correction is applied by the telescope's secondary mirror which is adaptive, the only one of its kind in the world.

- Wavefront sensing is done with a constellation of five laser guide stars. This is the only AO system to use more than one laser guide star.

- The laser beacons are generated by Rayleigh scattering of pulsed doubled-YAG laser beams. Although the MMT is not the first to use Rayleigh scattering, most AO systems use beacons generated by resonant backscatter of laser light tuned to the $589 \mathrm{~nm} \mathrm{D}_{2}$ resonance of atomic sodium in the mesosphere.

- The wavefront sensor includes a mechanism to track each laser pulse as it rises through the atmosphere at the speed of light, literally changing the focus of the telescope by moving a mirror in the optical train. This is done at the repetition rate of the laser, $5.2 \mathrm{kHz}$, and greatly extends the depth of field of the sensor.

- Uses: While not of direct relevance to the laser guide star program, the MMT AO system has been used to capture resolved images of geostationary satellites in the near IR (Figure 1).

Until very recently, AO systems supporting astronomical observing have used single guide stars to measure atmospheric turbulence. This has limited the best optical correction to a single target, with correction degrading as a function of

Adaptive Coded Aperture Imaging, Non-Imaging, and Unconventional Imaging Sensor Systems,

edited by Stanley Rogers, David P. Casasent, Jean J. Dolne, Thomas J. Karr, Victor L. Gamiz, Proc. of SPIE Vol. 7468, 74680L · C 2009 SPIE · CCC code: 0277-786X/09/\$18 · doi: 10.1117/12.827301 
increasing field angle. By using multiple guide stars, many types of wide field adaptive optics correction can be implemented. The simplest of these, ground-layer adaptive optics (GLAO), was suggested by Rigaut ${ }^{1}$ as a way to improve wide field imaging for large telescopes. Wavefront measurements from guide stars located far from each other (2-10 arc minutes) can be averaged to estimate the turbulence close to the telescope aperture. This component of the wavefront aberration is common to all objects over a wide field of view; when it is corrected by a single deformable mirror, the result is a partially corrected field that is as much as two orders of magnitude larger than the field achieved by extant AO systems.

GLAO can also be implemented using single Rayleigh laser guide star (LGS) systems at smaller telescopes. For example, the 4.2-m William Herschel Telescope has deployed one such a system ${ }^{2}$ and the 4.1-m Southern Astrophysical Research ${ }^{3}$ telescope is implementing another now. They are expected to produce wavefront compensation over fields of 1.5 and 3 arc minutes respectively. However, the laser light in all these cases does not come from infinity as starlight effectively does, and as the telescope diameter is increased, the mismatch in optical paths (the cone effect) eventually becomes so severe that no useful recovery of stellar wavefronts can be made. Hence a singlebeacon GLAO implementation will not be effective for larger apertures. ${ }^{4}$

Extremely large telescopes (ELTs) with diameters greater than $25 \mathrm{~m}$ will suffer the same problem even with higheraltitude sodium LGS. These telescopes therefore require a further advance in AO technique to exploit their full scientific potential. Multiple beacons placed so that their light collectively samples the full volume of atmosphere traversed by light from an astronomical target can in principle provide the solution. The instantaneous stellar wavefront would be computed by a tomographic algorithm applied to the wavefronts measured from all the beacons. ${ }^{5-7}$ This method, implemented with sodium beacon lasers, is planned for the Giant Magellan Telescope (GMT), ${ }^{8}$ and for the Thirty Meter Telescope, ${ }^{9}$ to recover the full resolution of their apertures.

\subsection{Scientific Potential for Ground-Layer Adaptive Optics Correction}

The key scientific breakthrough that will be exploited by the wide-field high resolution capability offered by GLAO correction is the realization of large near-infrared multi-object spectrographs (MOS) enabled by a new generation of multi-megapixel focal plane arrays. Two such instruments, called collectively LUCIFER, are being deployed at the Large Binocular Telescope (LBT). In Section 3.1 we describe our plans now underway to develop multi-laser GLAO at the LBT, building on the experience acquired at the MMT. LUCIFER will enable the LBT to undertake rapid spectroscopic surveys of large areas of the sky with dozens of objects examined in a single exposure. We give here just a few examples of the very broad range of astronomical science that will be enabled by the combination of GLAO and near infrared MOS:

- Sensitive stellar absorption line spectroscopy of massive galaxies at redshift $\sim 2$ will provide detailed constraints from key age-, star formation history-, and metallicity-sensitive diagnostic absorption features that are independent of interstellar dust reddening.

- High angular resolution multi-object emission line spectroscopy over a wide area (several arc minutes on a side) of substantial samples of galaxies with redshift $>1$. This will measure rotation curves for disk galaxies, determine velocity dispersions for early-type components and characterize the dynamics of the different components in interacting/merging systems.

- Quasar host galaxies: GLAO will be ground-breaking in making thousands of quasars accessible. Also, the pointspread function will be more stable over the field than that from classical AO correction because of the greatly reduced anisoplanatic wavefront error. 
- Black hole growth and cosmic reionization history from spectroscopy of quasars at very high redshift, $>6$. GLAO will greatly improve the efficiency of IR spectroscopy (in this case, of single objects) which measures quasar black hole masses and enables detections of inter-galactic medium absorption lines for reionization studies.

\section{THE MMT GLAO SYSTEM}

Simulations have predicted that multiple-beacon GLAO will effectively and consistently improve the atmospheric seeing, ${ }^{1,4,10,11}$ delivering $\sim 0.2$ arc second resolution in the near-infrared during a majority of seeing conditions. GLAO was first demonstrated on-sky in closed loop using three bright natural guide stars on a 1.5 arc minute diameter with the Multi-conjugate Adaptive optics Demonstrator (MAD) fielded at the Very Large Telescope (VLT) in early $2007 .^{12,13}$ However, sky coverage and the number of accessible science targets is severely limited with MAD because there are very few suitably bright natural guide star constellations.

\subsection{System Description}

The $6.5 \mathrm{~m}$ MMT in contrast deploys multiple laser beacons, Figure 2, to support wide-field science with GLAO. In addition, the AO system comprises an adaptive secondary mirror (ASM), a Cassegrain mounted wavefront sensor, and a PC based real-time reconstructor computer. The laser launch telescope, mounted above the MMT's ASM, projects a constellation of five $\lambda=532 \mathrm{~nm}$ beacons on a regular 2 arc minute diameter pentagon with a total on-sky power of $25 \mathrm{~W}$. The main characteristics of the system are summarized in Table 1.

Figure 3 provides a functional overview of the GLAO system which is essentially a closed-loop servo. Light from both the science field and the laser beacons enters the telescope. Science light (at wavelengths longward of $1.1 \mu \mathrm{m}$ ) passes through a dichroic beam splitter to the instrument. Visible light, including the LGS light, is reflected to a series of cameras. A second beam splitter, coated as a rugate filter to reflect a narrow band around the laser wavelength of $532 \mathrm{~nm}$, reflects the beacon light to a Shack-Hartmann sensor. The sensor divides the telescope pupil into 60 subapertures in a hexapolar geometry and puts the resulting spot patterns from all five LGS on a single CCD (Figure 4). Natural starlight is transmitted through the rugate filter to a tip-tilt sensor that measures rapid global image motion, not sensed by the LGS. A small fraction of the starlight is also directed to a second ShackHartmann sensor of high spatial order, $12 \times 12$ subapertures, but low temporal frequency, with a read rate of about once every $20 \mathrm{~s}$. This sensor is used to control non-common path errors between the LGS sensor and the science focal plane. Signals from all three cameras are fed to a fast computer that calculates the required commands to the ASM's actuators. The entire servo loop runs at an update rate of $400 \mathrm{~Hz}$.

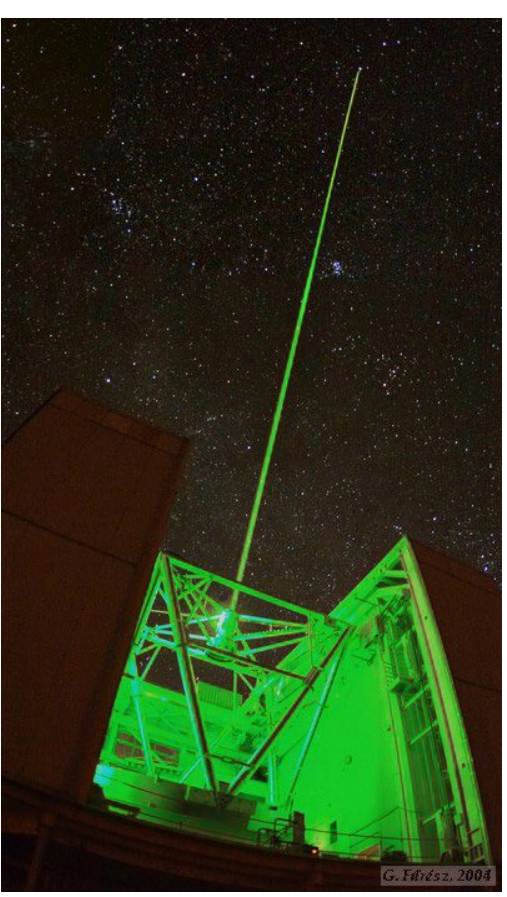

Figure 2. The MMT with the five RLGS beams being projected.

\begin{tabular}{|l|l|}
\hline Laser type & $2 \mathrm{x}$ doubled YAG (15 W each) \\
\hline Wavelength & $532 \mathrm{~nm}$ \\
\hline Pulse repetition rate & $5.2 \mathrm{kHz}$ \\
\hline Average power & $30 \mathrm{~W}$ \\
\hline Launch telescope location & Behind secondary mirror \\
\hline Number of beacons & 5, arranged as a regular pentagon \\
\hline Enclosed field of view & 2 arc minutes \\
\hline Beacon type & Rayleigh scattering \\
\hline Range gate & $20-29 \mathrm{~km}$ with dynamic refocusing \\
\hline
\end{tabular}

Table 1. Main characteristics of the multi-LGS system at the MMT. 


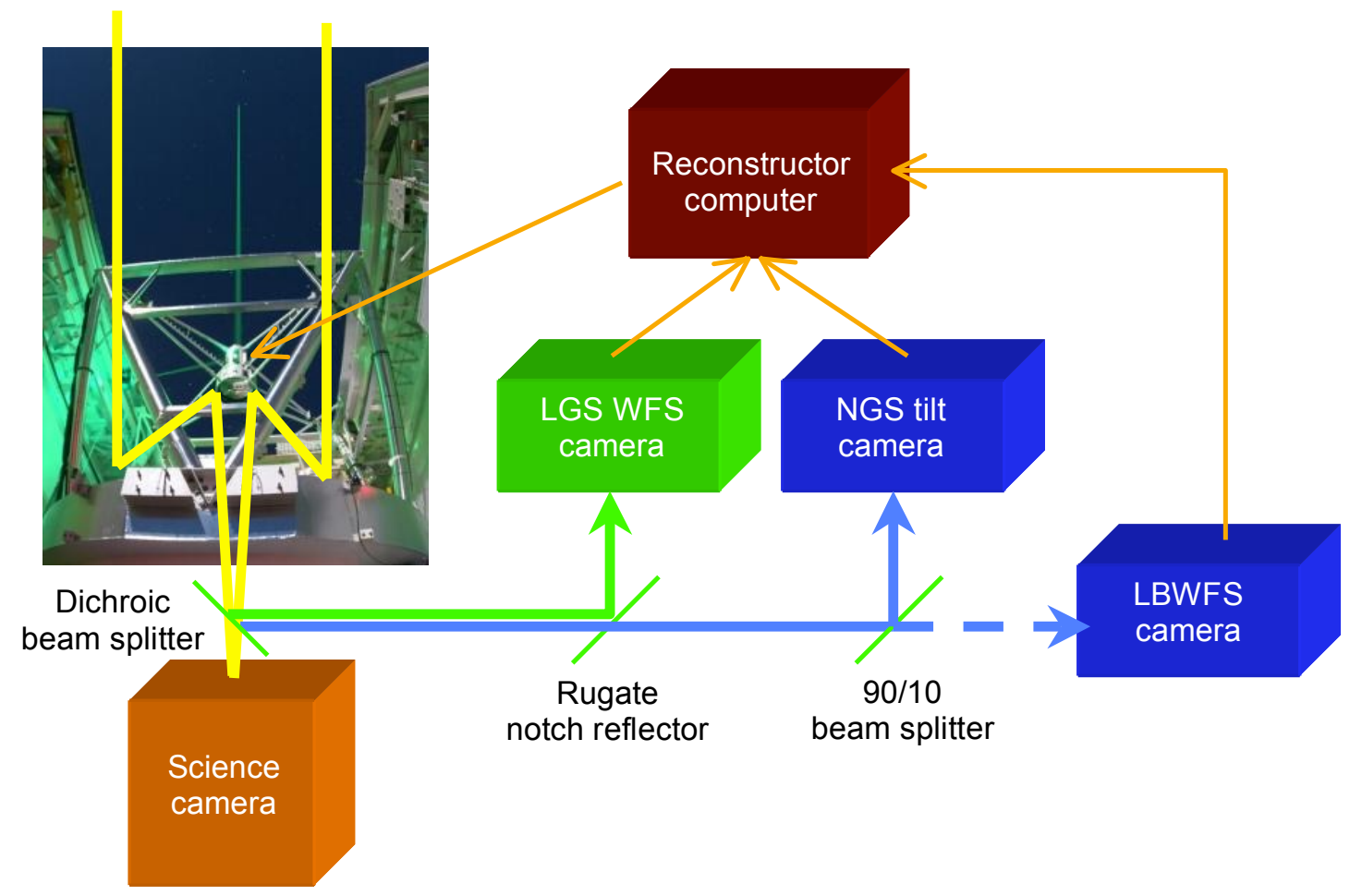

Figure 3. Flow of information, in the form of light and electronic signals, around the MMT's AO system. Light from the science target and the laser beacons enters the telescope. IR light passes through a dichroic beam splitter to the science camera while visible light from the RLGS and the tip-tilt star is reflected to the wavefront sensing cameras. Output from the cameras is used by a real-time control system to drive the actuators of the telescope's ASM. Further details are given in the text.

The lasers emit short pulses at $5.2 \mathrm{kHz}$ rep rate. In order to capture enough of the back-scattered return from each pulse, the optical system is dynamically refocused at the same frequency. This is done by means of a mirror mounted on a mechanical resonator. The resonator acts as the master clock for the system, with the timing of the laser pulse firing and the operation of the shutter in the wavefront sensor camera tied to its motion with programmable phase delays.

\subsection{Closed-Loop Results}

The MMT system is now working routinely to give image quality of $\sim 0.25$ arc sec in $\mathrm{K}$ band $(2.2 \mu \mathrm{m})$ over the 2 arc min field spanned by the LGS constellation. The scientific instrument used is PISCES, an imaging camera in the near infrared (1.2-2.5 $\mu \mathrm{m})$ with 110 arc sec field of view and 107 mas pixels. Observations of the globular cluster M3 show the full potential for GLAO. Figure 5 shows $60 \mathrm{~s}$ images of the cluster core in $\mathrm{K}$ band. In this instance, the seeing of 0.7 arc sec was reduced only to $0.30 \mathrm{arc} \mathrm{sec}$, yet the better resolution leads to an improved detectability of 2 magnitudes in this very crowded region.

We have examined the behavior of the point-spread function (PSF) as a function of field angle from observations of the open Galactic star cluster M34. ${ }^{15}$ Figure 6 shows the measured FWHM: the mean uncorrected value was 0.86 arc sec (median for $\mathrm{K}$ band is 0.60 arc sec) which improved to 0.27 arc sec with GLAO correction. . The reduction in width represents an improvement in seeing from poor to better than the 10th

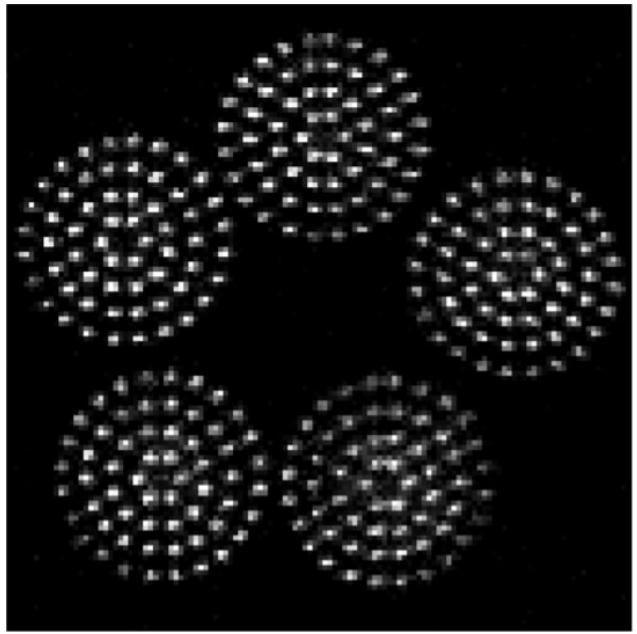

Figure 4. Single frame from the RLGS wavefront sensor camera showing the 60 spot patterns generated by each of the five laser beacons. 

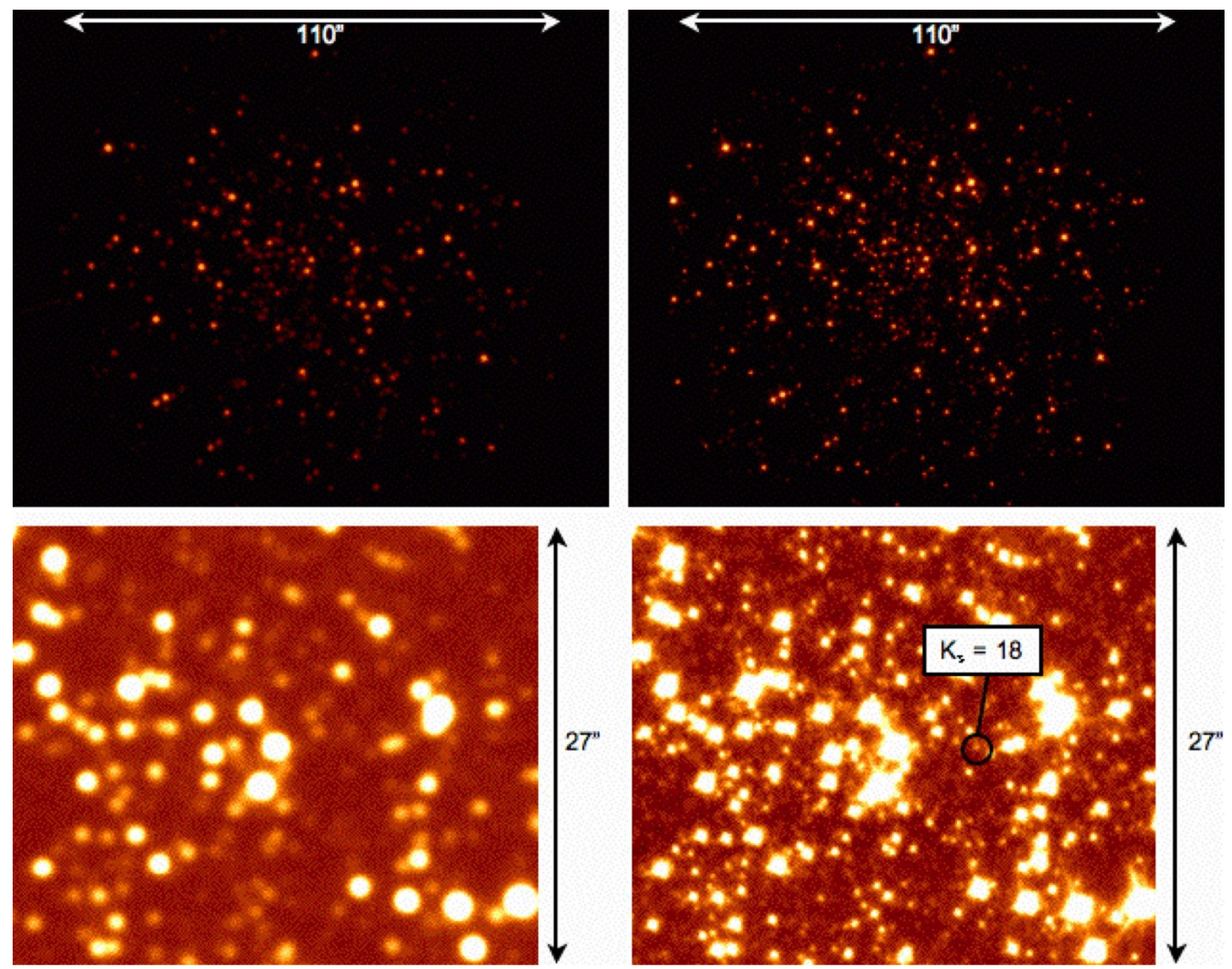

Figure 5. The core of M3 imaged in K band in two 60-s exposures. (Top) The full field of PISCES shown on a logarithmic stretch, in the seeing limit (left) and with GLAO running at $400 \mathrm{~Hz}$ (right). (Bottom) Zoomed portions of the upper images shown on a truncated linear scale that reaches the noise floor. For reference, a star with $\mathrm{m}_{\mathrm{K}}=18$ is shown in the corrected image, detected at SNR of 7 . In the uncorrected image stars must be 2 magnitudes brighter to be detected at the same SNR.

percentile for the site. Furthermore, of particular importance to spectroscopy which is improved with higher energy concentration, the peak intensity increased by a factor of 5.5 and the encircled energy flux within a 0.2 arc sec circular aperture increased by a factor of 3.8 .

Images of another open cluster, M36, were recorded in $\mathrm{J}, \mathrm{H}$ and $\mathrm{K}$ filters $(1.25 \mu \mathrm{m}, 1.65 \mu \mathrm{m}$, and $2.2 \mu \mathrm{m}$ center wavelengths respectively). Average radial profiles for 60-s exposures of stars chosen from across the field were computed and are shown in Figure 7. Image sharpening is as expected best in the $\mathrm{K}$ band, which improved from 0.65 arc sec to 0.22 arc sec, but remarkably, the system is effective at correcting at $\mathrm{J}$ band to 0.29 arc sec. This had not been expected; the best performance predicted by numerical modeling at this shorter wavelength being around 0.4 arc sec for these approximately median conditions. The result is arguably attributable to an unusually low and thin ground layer, or a high ratio between ground layer and free atmosphere turbulence, but good J-band performance has now been observed during several telescope runs under a variety of seeing conditions at different times of the year.

We have also modeled the K-band PSF width as a function of the V magnitude of the tip-tilt star in average seeing conditions. The model is based on measurements recorded at the MMT using a $10^{\text {th }}$ magnitude tip-tilt star with variable amounts of neutral density filtering inserted before the wavefront sensor (WFS). The model assumes a K5 main 


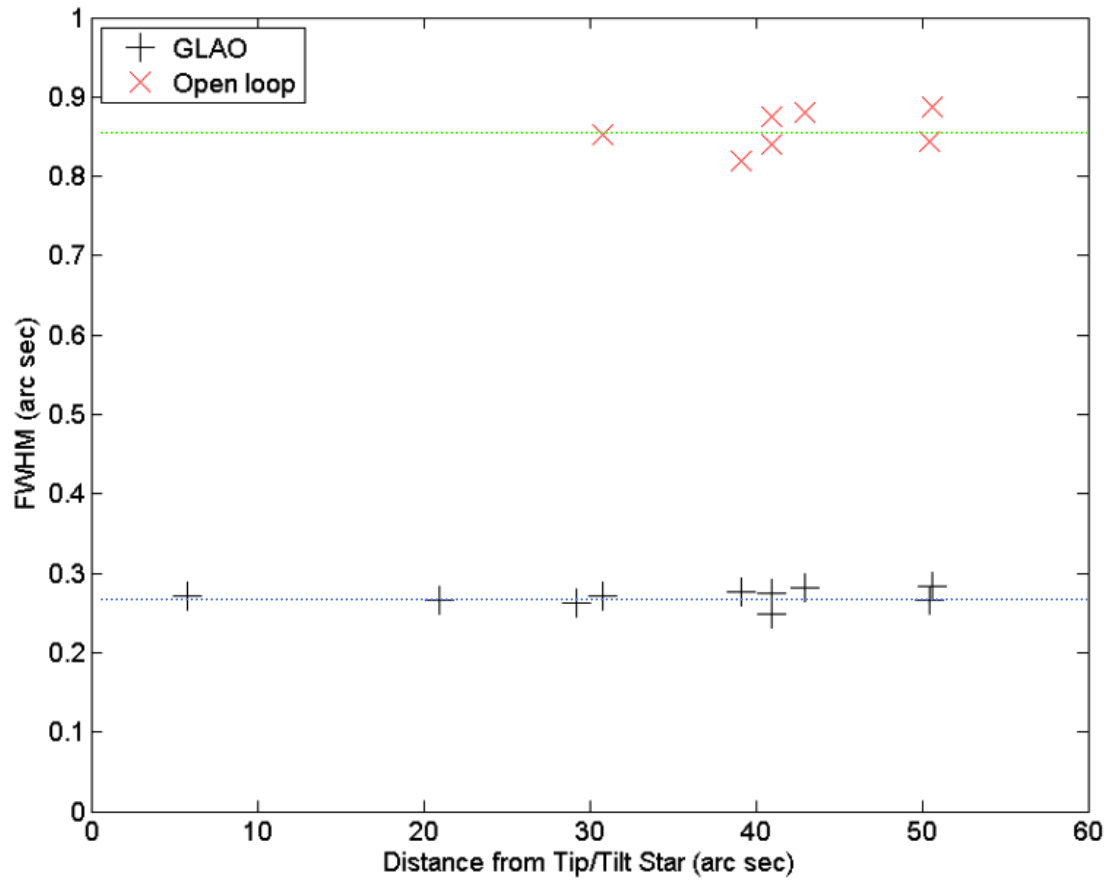

Figure 6. Comparison of open loop and closed loop FWHM vs. distance from the tip-tilt star across the 2 arc min PISCES field for M34 at $\lambda=2.14 \mu \mathrm{m}$ using GLAO correction. The horizontal dotted lines represent the K-band seeing limit and average corrected width across the field. Plus symbols indicate closed loop and X symbols indicate open loop measurements.

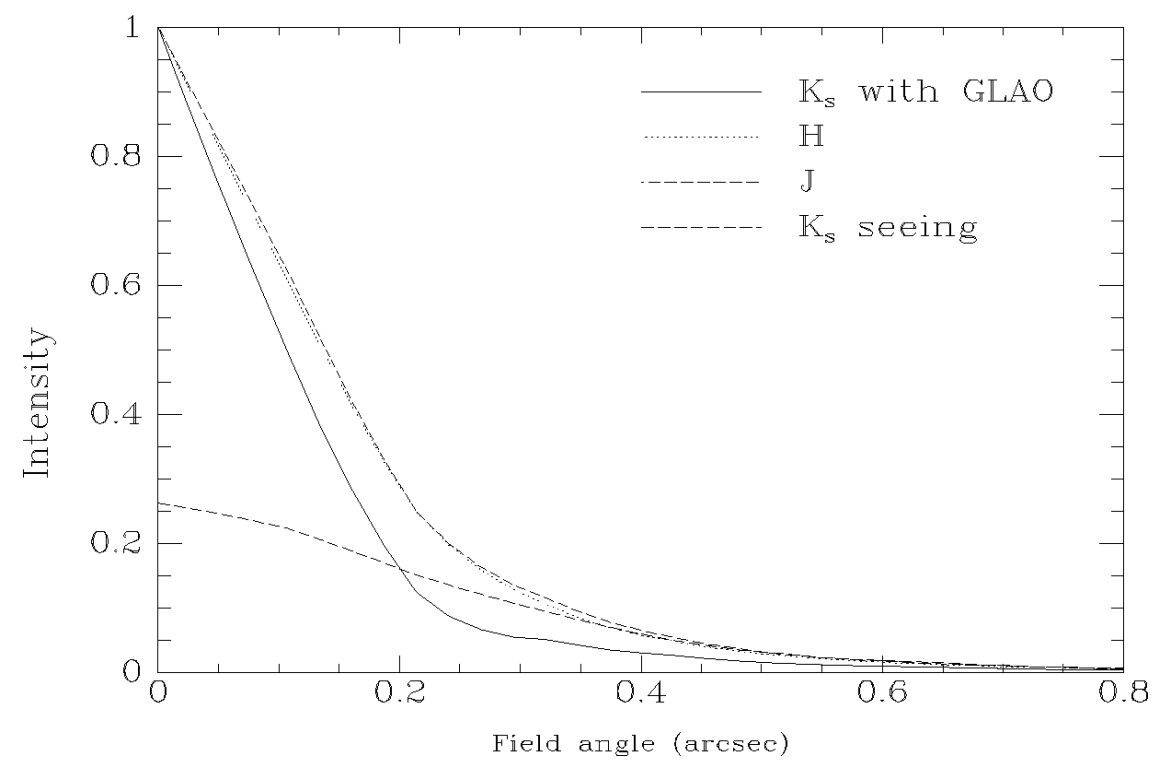

Figure 7. Radial profiles of the GLAO-corrected point-spread functions, normalized to unit peak intensity, in the $\mathrm{J}(1.25 \mu \mathrm{m}), \mathrm{H}(1.65 \mu \mathrm{m})$, and $\mathrm{K}(2.2 \mu \mathrm{m})$ wavebands. The FWHM are respectively $0.29,0.29$, and 0.22 arc sec. Also shown for comparison is the profile of the seeing-limited K-band PSF, normalized to the same total energy as the corrected K-band PSF. The FWHM is 0.63 arc sec, slightly worse than median for the site. 
sequence star, the median type found for wavefront sensing, and a broad-band EMCCD tip-tilt sensor. As shown in Figure 8, full performance will be maintained down to $\mathrm{V}$ magnitude 18 with graceful degradation for fainter stars to around $21^{\text {st }}$ magnitude; this provides about $80 \%$ sky coverage. In contrast, the performance of diffraction-limited AO systems drops off more rapidly as the high-resolution image disintegrates into a speckle cloud.

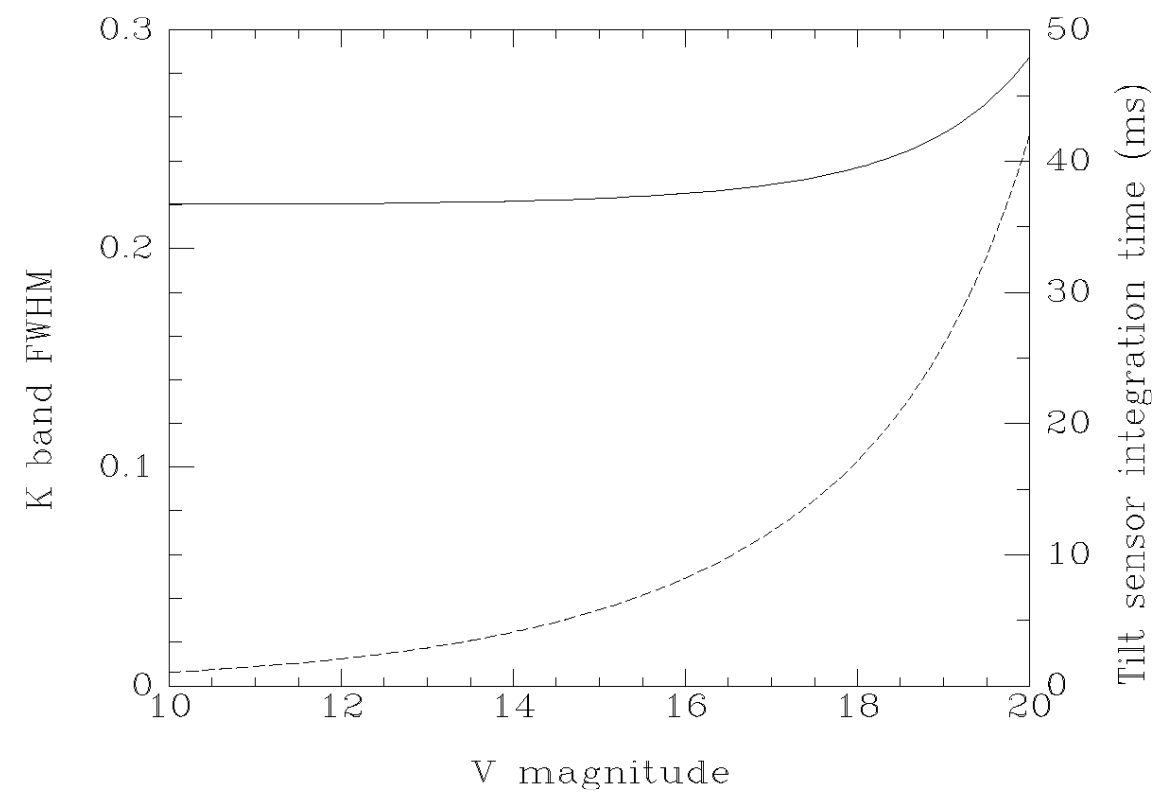

Figure 8. Anticipated performance of the MMT GLAO system under average seeing conditions, expressed as the FWHM of the delivered K-band PSF, as a function of the brightness of the tip-tilt star in the V band (solid line). Also shown by the dashed line is the optimal integration time on the tip-tilt sensor which balances photon noise and servo lag in the residual uncorrected wavefront error. Full performance is maintained to magnitude 18 with graceful degradation of performance for fainter stars.

\section{EXTENSION TO FUTURE LARGE TELESCOPES}

\subsection{GLAO for the Large Binocular Telescope}

The LBT, shown in Figure 9, in which the University of Arizona is a $25 \%$ partner, is now the single largest optical/infrared telescope in the world and for this and other reasons offers uniquely powerful scientific capabilities. In particular, the LBT was designed from the outset with AO correction built-in to the telescope's secondary mirrors, developed from the successful design of the MMT's ASM. The LBT is a Gregorian design, meaning that the concave ASMs are optically conjugated about $100 \mathrm{~m}$ above the telescope, in the middle of the ground-layer turbulence. The design makes these mirrors ideal for correcting the low altitude atmospheric wavefront aberration over a wide field of view. No other $8 \mathrm{~m}$-class telescope has this capability, which allows for correction of all instruments used at the Gregorian foci, without any requirement for wide field relay optics that introduce losses and increase thermal background, complexity, and cost.

A GLAO system that also builds on the work at the MMT is

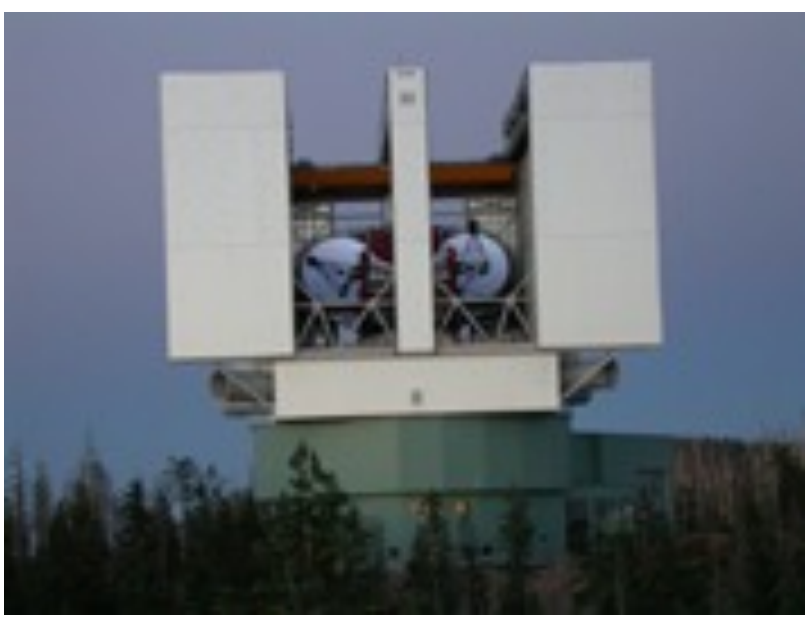

Figure 9. The Large Binocular Telescope atop Mt. Graham in Arizona. Each of the two primary mirrors is $8.4 \mathrm{~m}$ in diameter and the telescope is being equipped with adaptive secondary mirrors. 
now in the advanced design stage, having passed a Preliminary Design Review in February 2009. The Advanced Rayleigh Ground layer adaptive Optics System (ARGOS) will deploy three Rayleigh LGS gated at $12 \mathrm{~km}$ range above each half of the LBT, to correct a 4 arc min field of view. Each half of the LBT will be corrected independently with no attempt to cophase them. Image quality at the expected level of 0.2 arc sec will be fully exploited by the wide-field imaging and multi-object spectrographic modes of the two LUCIFER instruments, one deployed on each eye of the LBT. Each instrument can operate in a number of modes: a) wide-field imaging with 0.12 arc sec sampling over a $4 \times 4$ arc min field; b) cold-slit multi-object spectroscopy over a $2 \times 4$ arc min field with spectral resolution $\mathrm{R} \sim 3000$; c) long-slit single-object spectroscopy with $\mathrm{R} \sim 10,000$; d) a narrow field imaging mode with 15 mas pixels over a $30 \times 30$ arc sec field.

The first LUCIFER has been delivered to the telescope and has undergone initial tests on sky. Figure 10 shows the raw first-light K-band image, when the seeing was very good; the stellar PSFs have a FWHM of 0.3 arc sec. While this shows the excellence of the LBT site, seeing this good occurs only about $10 \%$ of the time. With ARGOS, we can expect to achieve image quality at least this good more than half the time. The system is expected to be fully operational by the end of 2011 .

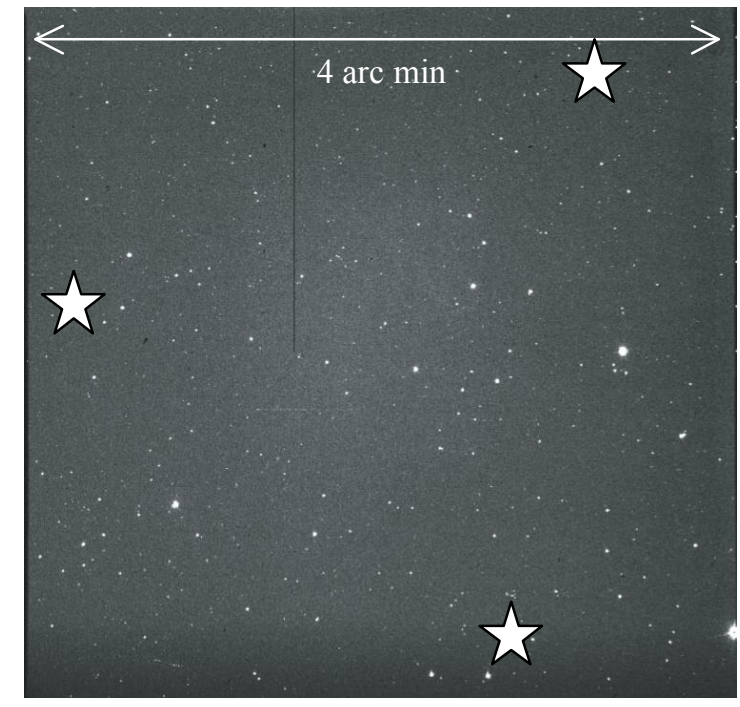

Figure 10. First light image from the first LUCIFER. This image shows a 4 arc min region near HD 333240 in $\mathrm{K}$ band. PSF width is 0.3 arc sec. The star symbols show the approximate locations in the field of the three future LGS.

The ARGOS system is designed to be upgraded in two further phases that will deliver the diffraction limit over the conventional narrow field, as well as GLAO. In Phase II, a sodium LGS will be added to be launched with the Rayleigh beacons coaxially with each primary mirror. With the Rayleigh and sodium LGS working together, high-quality diffraction-limited imaging will be enabled at wavebands at least as short as $1.2 \mu \mathrm{m}$, and will be exploited by the narrow field mode of LUCIFER. In a further extension of capability, the addition of a second set of wavefront sensors for the Rayleigh LGS will allow the LBT Interferometer ${ }^{15}$ (Hinz et al. 2003) to take advantage of the AO correction to reach the diffraction limit of the coherently combined apertures in $\mathrm{L}$ and $\mathrm{M}$ bands over most of the observable sky. In Phase III, a near-IR imaging interferometer called LINC-Nirvana ${ }^{16}$ (Egner et al. 2004) will be equipped with an additional deformable mirror conjugated to high altitude turbulence in each of the two beams, bringing the full power of the hybrid LGS system for tomography to bear to provide multi-conjugate AO. Wide-field near IR imaging at the $22.8 \mathrm{~m}$ diffraction limit will thereby be enabled.

\subsection{Multi-laser adaptive optics for the Giant Magellan Telescope}

The Giant Magellan Telescope (GMT, Figure 11) is an international partnership to build a $25 \mathrm{~m}$ telescope using seven $8.4 \mathrm{~m}$ primary mirror segments. It relies for much of its technology on techniques proven on the LBT and MMT. The primary mirror segments are the same size as those of the LBT, and, like the LBT, the adaptive optics will be directly built into the telescope with a concave Gregorian ASM. The secondary mirror will be built as seven discrete circular segments, each $1.06 \mathrm{~m}$ in diameter, matched one-for-one with the primary mirror segments. The GMT will be sited on Cerro Las Campanas in Chile. The first of the seven primary mirror segments has now been cast at the University of Arizona's Mirror Lab.

GMT and other extremely large telescopes now being planned ${ }^{17,18}$ will be very costly scientific instruments. It is essential then that the scientific return on the investment be maximized by operating these telescopes at high resolution with AO. The first-light AO system designed for the GMT will allow for the use of natural guide stars when one happens to be present, but will also field six sodium laser guide stars in a regular hexagon with variable size. With the beacons at $\sim 6$ arc min diameter, GLAO will be implemented in a manner very similar to the current system on the MMT, with the mean of the six beacon wavefront measurements being used to compute the correction to be applied to the ASM. Because of the Gregorian configuration which puts the image of the ASM as seen by the primary mirror at about $150 \mathrm{~m}$ above the telescope, within the boundary layer, the GMT is particularly well suited to GLAO over a wide field. 


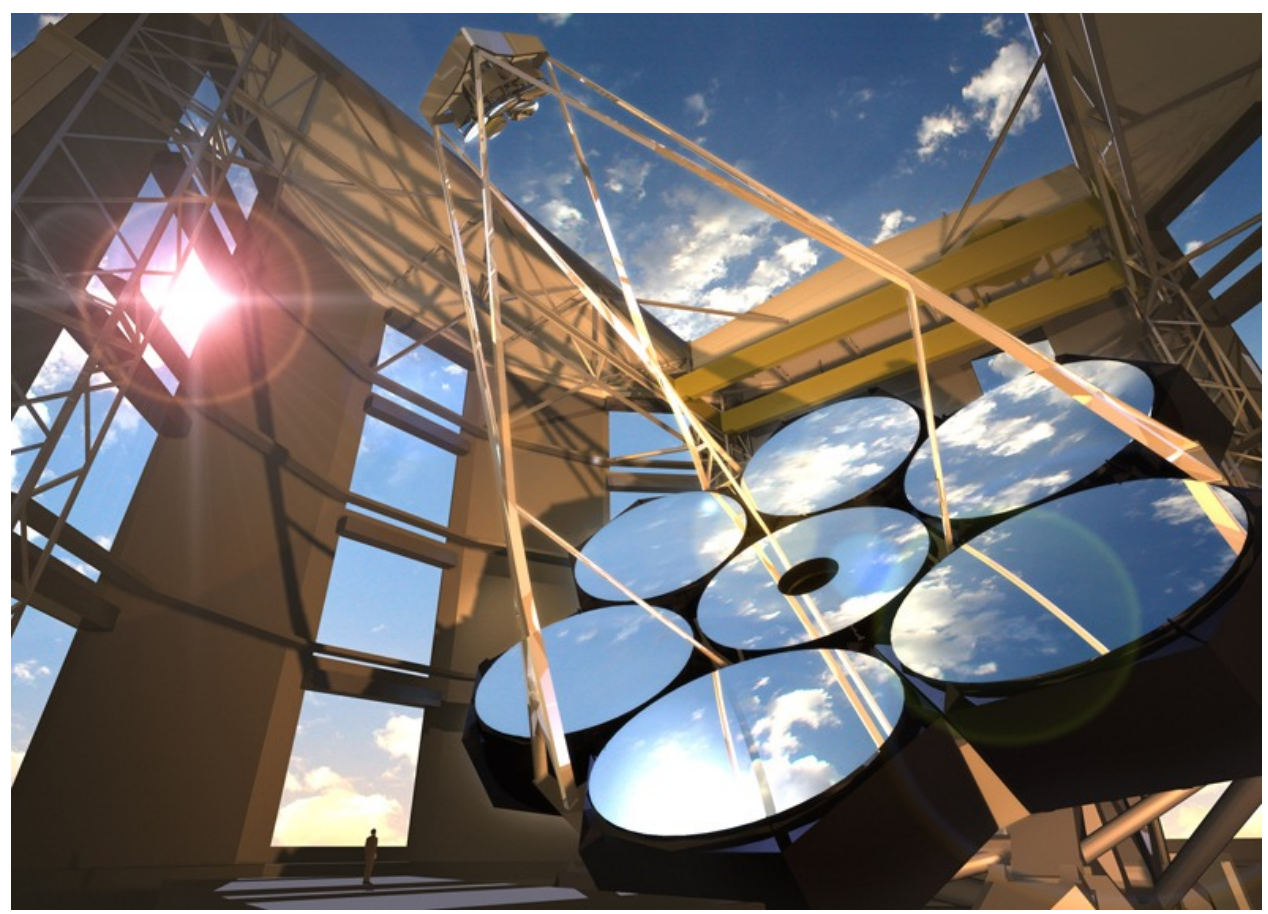

Figure 11. Artist's conception of the $25 \mathrm{~m}$ diameter Giant Magellan Telescope which will comprise seven $8.4 \mathrm{~m}$ mirror segments on a common mount. The first of the off-axis segments is now in production at the University of Arizona's Mirror Lab and the whole telescope will be built atop Cerro Las Campanas in the Chilean Andes.

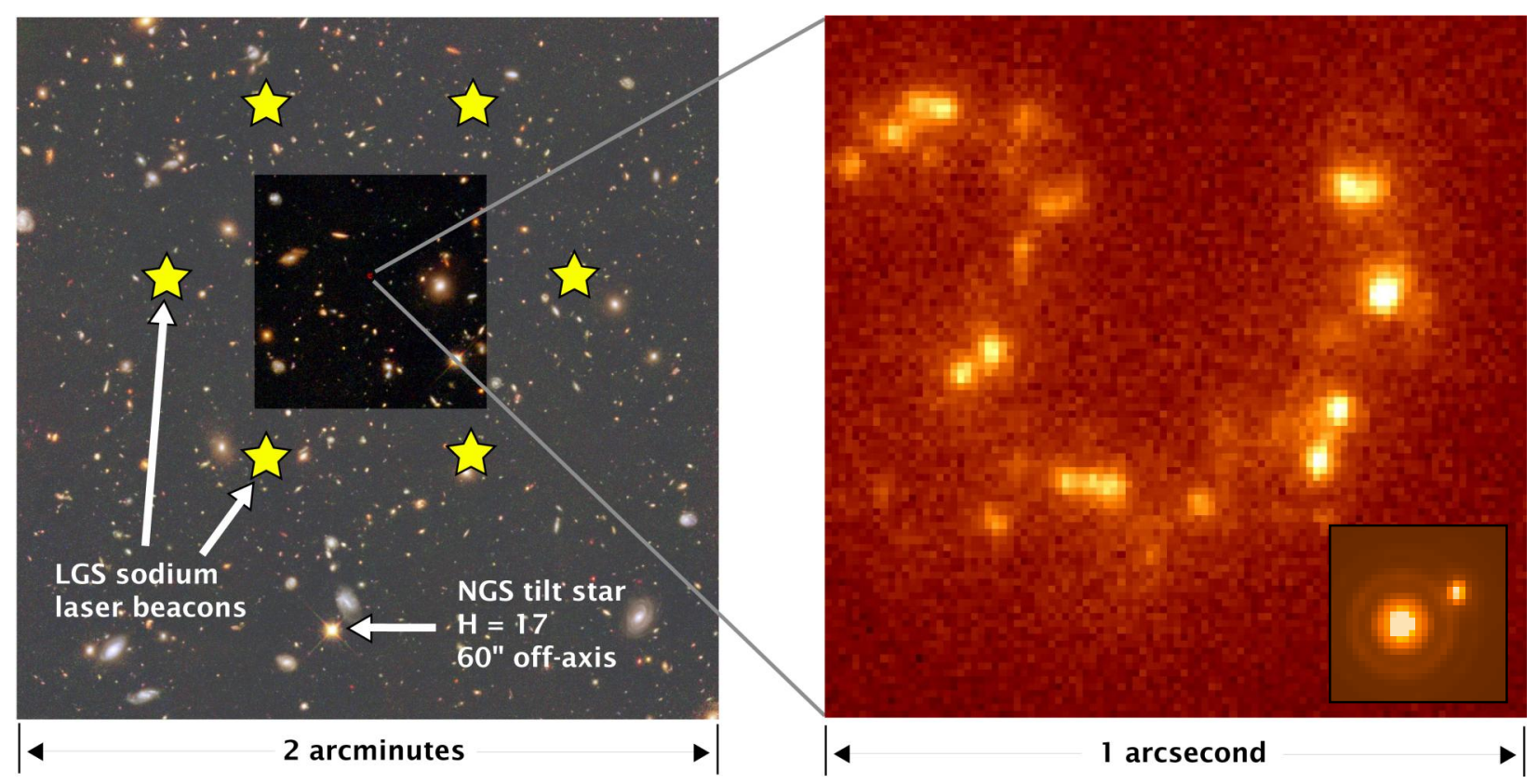

Figure 12. (Right) A simulated one hour GMT image of a starburst galaxy at redshift 1.4, demonstrating the GMT's 0.016 arc sec $\mathrm{H}$ band resolution with adaptive optics, ten times the resolution of HST. This simulation is based on an Ha image of NGC 3089 at a detector plate scale of 0.010 arc sec per pixel. This narrow band image is centered at the redshifted $\mathrm{H} \alpha$ wavelength of $1.58 \mu \mathrm{m}$. (Left) The full field of the GMT AO imager is $40 \mathrm{arc}$ sec across, with an area 1600 times larger than the detail to the right. The positions of the constellation of laser guide star (LGS) beacons and the natural guide star (NGS) used to recover tip-tilt information are indicated 
Diffraction-limited imaging in the near and thermal infrared $(1.2-10 \mu \mathrm{m})$ will be enabled by reducing the laser constellation diameter to $35 \mathrm{arc} \mathrm{sec}$ and applying a tomographic analysis to the beacon signals. In this way, rather than recovering the aberration in a single horizontal layer, as is the case with GLAO, the full 3-D structure of the aberration is reconstructed, allowing the line-of-sight integral in the direction of a particular science target to be estimated and corrected with the ASM.

Figure 12 shows a simulated example of correction by this means. The 40 arc sec square field of the GMT's planned HRCam imager is shown on the left superposed on a deep 2 arc min square NICMOS field from the HST. Simulated $\mathrm{AO}$ correction over this field is made from wavefront measurements of the six laser beacons and a guide star 1 arc min off axis of $\mathrm{H}$ magnitude 17. On the right is a detail of a 1 arc sec square within the corrected field showing a starburst galaxy at redshift 1.4, as it would be detected in an $\mathrm{H}$ band image with the 16 mas resolution of the GMT AO system, ten times that of HST. The input was an $\mathrm{H} \alpha$ image of NGC 4038/39. The individual redshifted HII regions were taken to have $\mathrm{H}$ magnitude $\sim 24.5$. The detector plate scale is $10 \mathrm{mas} / \mathrm{pixel}$. Sky noise is modeled for a 1 hour exposure through a $2.5 \%$ filter at the redshifted $\mathrm{H} \alpha$ wavelength. The insert shows the stellar PSF with the same 10 mas pixel size and the projected $40 \%$ Strehl ratio for two stars, one saturated, separated by 100 mas. The elongation is due to increased tilt anisoplanatism in the direction of the guide star.

\section{CONCLUSIONS}

The multiple LGS AO program at the MMT is exploring the practical techniques required for wavefront sensing for ground-layer and in the immediate future will be used also to investigate tomographic correction. Closed-loop GLAO operation has been successfully demonstrated with resolution in the near IR improved routinely from $0.6-0.8$ arc sec to $0.2-0.3$ arc sec over a wide field of 2 arc min diameter. Future plans with the MMT call for additional existing near- and thermal-IR instruments to be mated to the GLAO system to provide imaging over a broader range of wavelength coverage, and near-IR spectroscopy.

The application of GLAO to near-IR multi-object spectroscopy in particular will be a powerful tool enabling new kinds of astronomical science. This is the motivation behind the development of the ARGOS ground-layer system for the LBT. Studies of the early universe, which require large statistically significant samples of faint objects at high redshift, will benefit the most from the new technology. It is impractical with contemporary AO systems to make large surveys of such objects in a reasonable time because of the small fields of view to which such systems are limited. GLAO on the other hand will open up much bigger regions of the sky to high-resolution imaging.

Once ARGOS is upgraded to diffraction-limited imaging with tomography, the LBT with its $23 \mathrm{~m}$ baseline will become a unique trail-blazer and the first of the new generation of extremely large telescopes. All the ELT projects now in development in the US and Europe will require multi-laser AO for all-sky access at full resolution. Single lasers will no longer suffice, being limited by focal anisoplanatism even at the $90 \mathrm{~km}$ range of a sodium beacon. ARGOS will allow exploration and evaluation of the complex range of techniques required to realize the power of these giant and very expensive future telescopes, as well as give astronomers their first data at ELT resolution.

Observations reported here were made at the MMT, a joint facility of the University of Arizona and the Smithsonian Institution. This work has been supported by the National Science Foundation under grant AST-0505369.

\section{REFERENCES}

[1] Rigaut, F., 2002, "Ground-conjugate wide field adaptive optics for the ELTs," in Beyond Conventional Adaptive Optics, ed. E. Vernet, et al., Proc. ESO 58, 11-16.

[2] Tokovinin, A., et al., 2008, "SAM - a facility GLAO instrument," in Adaptive Optics Systems, eds. N. Hubin, C. Max \& P. Wizinowich, Proc. SPIE 7015.

[3] Martin, O., et al., 2008, "Optomechanical commissioning of the GLAS Rayleigh laser guide star for the WHT," in Adaptive Optics Systems, eds. N. Hubin, C. Max \& P. Wizinowich, Proc. SPIE 7015.

[4] Andersen, D., et al., 2006, "Performance Modeling of a Wide-Field Ground-Layer Adaptive Optics System," Publ. Astron. Soc. Pac. 118, 1574-1590.

[5] Ragazzoni, R., Marchetti, E., \& Rigaut, F., 1999, A\&A, 342, L53

[6] Tokovinin, A., Le Louarn, M., Viard, E., Hubin, N., \& Conan, R., 2001, A\&A, 378, 710

[7] Lloyd-Hart, M., \& Milton, N. M., 2003, JOSA A, 20, 1949 
[8] Milton, N. M., Lloyd-Hart, M., Cheng, A., Georges, J., \& Angel, J. R. P., 2003, in Astronomical Adaptive Optics Systems and Applications, eds. R. K. Tyson \& M. Lloyd-Hart, Proc. SPIE 5169, 238

[9] Dekany, R. G., Britton, M. C., Gavel, D. T., Ellerbroek, B. L., Herriot, G., Max, C. E., and Veran, J.-P., 2004, in Advancements in Adaptive Optics, eds. D. Bonaccini, B. L. Ellerbroek, \& R. Ragazzoni, Proc. SPIE 5490, 879

[10] Le Louarn, M. and Hubin, N., 2006, "Improving the seeing with wide-field adaptive optics in the near-infrared," Mon. Not. R. Astron. Soc. 365, 1324-1332.

[11] Tokovinin, A., 2004, "Seeing improvement with ground-layer adaptive optics," Publ. Astron. Soc. Pac. 116, 941951.

[12] Marchetti, E., et al., 2007, "On-sky Testing of the Multi-Conjugate Adaptive Optics Demonstrator," The Messenger 129, 8-13.

[13] Bouy, H., et al., 2008, "Multi conjugate adaptive optics images of the Trapezium cluster," A\&A 477, 681-690.

[14] Baranec, C., et al., 2009, "On-sky wide field adaptive optics correction using multiple laser guide stars at the MMT," ApJ, 693, 1814.

[15] Hinz, P. M. et al., 2003, "The Large Binocular Telescope Interferometer," in Interferometry for Optical Astronomy II, ed. W. A. Traub, Proc. SPIE 4838, 108.

[16] Egner, S. E. et al., 2004 "LINC-NIRVANA: the single arm MCAO experiment," in Advances in Adaptive Optics, eds. D. Bonaccini, B. L. Ellerbroek, and R. Ragazzoni, Proc. SPIE 5490, 924.

[17] Nelson, J. and Sanders, G., 2008, "The status of the Thirty Meter Telescope project," in Ground-based and Airborne Telescopes II, eds. L. M. Stepp \& R.Gilmozzi, Proc. SPIE 7012.

[18] Gilmozzi, R. and Spyromillio, J., 2008, "The 42m European ELT: status," in Ground-based and Airborne Telescopes II, eds. L. M. Stepp \& R.Gilmozzi, Proc. SPIE 7012. 